\title{
The effect of chronic alcohol intoxication and smoking on the activity of oral peroxidase
}

\author{
Napoleon Waszkiewicz ${ }^{1}$, Anna Zalewska ${ }^{2}$, Sławomir Dariusz Szajda ${ }^{3}$, Agata Szulc ${ }^{1}$, \\ Alina Kępka ${ }^{4}$, Alina Minarowska ${ }^{5}$, Marzena Wojewódzka-Żelezniakowicz ${ }^{3}$, \\ Beata Konarzewska $^{1}$, Sylwia Chojnowska ${ }^{6}$, Zbigniew Bronisław Supronowicz ${ }^{7}$, \\ Jerzy Robert Ladny ${ }^{3}$, Krzysztof Zwierz ${ }^{8}$ \\ ${ }^{1}$ Department of Psychiatry, Medical University of Bialystok, Poland \\ ${ }^{2}$ Department of Pedodontics, Medical University of Bialystok, Poland \\ ${ }^{3}$ Department of Emergency Medicine and Disasters, Medical University of Bialystok, Poland \\ ${ }^{4}$ Department of Biochemistry and Experimental Medicine, the Children's Memorial Health Institute, \\ Warsaw, Poland \\ ${ }^{5}$ Department of Anatomy, Medical University of Bialystok, Poland \\ ${ }^{6}$ Medical Institute, College of Computer Science and Business Administration, Lomza, Poland \\ ${ }^{7}$ Department of Detoxification, Psychiatric Hospital, Choroszcz, Poland \\ ${ }^{8}$ Medical College of the Universal Education Society, Lomza, Poland
}

\begin{abstract}
Peroxidase is the most important antioxidant enzyme in saliva. Through peroxidation of thiocyanate in the presence of $\mathrm{H}_{2} \mathrm{O}_{2}$, peroxidase catalyses the formation of bacteriocidic compounds such as hypothiocyanate. The purpose of this study was to evaluate the effect of chronic alcohol intoxication and smoking on the activity of oral peroxidase (OPO). A total of 37 volunteers participated in the study. This cohort consisted of 17 male alcohol-dependent smoking patients after chronic alcohol intoxication (AS group, alcohol + smoking) (mean age: 42 years; range: 26-55) (100-700 g/day of alcohol; 10-20 cigarettes/day) and 20 control male social drinkers (CNS group, control non-smokers) with no history of alcohol abuse or smoking (mean age: 42 years; range: 30-53). Salivary peroxidase activity was measured by the colorimetric method. The differences between groups were evaluated using the Mann-Whitney U test. There was significantly higher activity of OPO $(p=0.00001)$ and significantly lower salivary flow $(\mathrm{SF})(\mathrm{p}=0.007)$ in alcohol-dependent smokers after chronic alcohol intoxication compared to the control group. OPO activity significantly correlated with the number of days of alcohol intoxication, but not with smoking. Gingival index (GI) was significantly higher in smoking alcohol-dependent persons than in the control group, and correlated with OPO activity. The sensitivity of the OPO test was $70 \%$ in smoking alcoholics, while specificity was $95 \%$. The increased activity of OPO suggests chronic oxidative stress is more likely due to ethanol action than to smoking. Smoking alcohol-dependent persons have a worse periodontal status than controls. OPO activity as a marker of chronic alcohol abuse may help in the diagnosis of alcoholism. (Folia Histochemica et Cytobiologica 2012, Vol. 50, No. 3, 450-455)
\end{abstract}

Key words: alcohol, smoking, saliva, oral peroxidase

Correspondence address: N. Waszkiewicz,

Department of Psychiatry, Medical University of Bialystok, Plac Brodowicza 1, 16-070 Choroszcz, Poland;

tel./fax: + 48857193977 ;

e-mails: napoleonwas@yahoo.com,napwas@wp.pl

\section{Introduction}

It is estimated that $3.8 \%$ of all global deaths, $4.6 \%$ of the global burden of disease and injury, and $30 \%$ of all hospital admissions and healthcare costs are at- 
tributable to alcohol abuse [1]. About $2 \%$ of the population suffers from alcohol dependence. During drinking, ethanol diffuses rapidly into saliva, and immediately after drinking its salivary concentration is temporarily much higher than in plasma. Within 30 minutes, salivary ethanol concentration equilibrates with the plasma level, thus suggesting that ethanol penetrates the whole body, including the salivary glands. After alcohol intake, the level of acetaldehyde in saliva strikingly exceeds the level in systemic blood. From saliva, acetaldehyde and ethanol easily reach all local tissues. Damage to the oral tissues seems to be mostly attributable to the action of acetaldehyde, although some acute effects depend on the direct action of ethanol, the formation of reactive oxygen species (ROS), non-oxidative metabolites of ethanol (e.g. fatty acid ethyl esters - FAEEs), and the ethanolwater competition mechanism [2-9].

Acetaldehyde, the main metabolite of ethanol, is also a component of tobacco smoke and has been identified as an inducer of carcinogenesis [10]. In addition to acetaldehyde, tobacco smoke contains as many as 3,000 toxic substances such as nicotine, nitrosamines, carbon monoxide, etc. [11]. Cigarette smoking and alcohol drinking often co-occur [12], and are highly correlated even if they occur occasionally [13]. Acetaldehyde destroys enzymes involved in the defense of oral tissues such as peroxidase [14]. Even in persons who use snuff or chew tobacco, decreases in the levels of lysozyme and lactoferrin, and an increase in the salivary IgA, have been found [15]. Chronic alcohol abuse and cigarette smoking have been found to decrease protein secretion with accompanying reduction in the level of amylase in saliva $[6,15]$.

Peroxidase is the most important antioxidant enzyme in saliva. Peroxidase is a glycoprotein, the activity of which has been found in many exocrine secretions such as tears, milk, vaginal secretions and saliva $[5,16]$. Oral peroxidase (OPO) has a dual origin: salivary peroxidase (SPO) is produced and secreted by salivary gland cells, and myeloperoxidase (MPO) is produced by polymorphic neutrophils (PMN) that migrate into the oral cavity with gingival crevicular fluid [17]. Salivary peroxidase (SPO) represents $60-80 \%$ of the total OPO activity [18]. Myeloperoxidase forms 20 to $40 \%$ of the total OPO activity, gaining in importance in inflammatory conditions (30$70 \%)[17,18]$. OPO has a dual role: it controls the level of toxicity of hydrogen peroxide $\left(\mathrm{H}_{2} \mathrm{O}_{2}\right)$ excreted by bacteria and leukocytes, and it also exhibits a specific antibacterial activity by inhibiting the metabolism and proliferation of $\mathrm{G}(-)$ and $\mathrm{G}(+)$ bacteria in the mouth $[19,20]$. Through peroxidation of thiocyanate in the presence of $\mathrm{H}_{2} \mathrm{O}_{2}$, peroxidase catalyses the formation of bacteriocidic compounds, e.g. hypothiocyanite, which inhibit bacterial metabolism (i,e. react with sulfhydryl groups of the enzymes involved in glycolysis) $[15,19,21,22]$. The use of $\mathrm{H}_{2} \mathrm{O}_{2}$ by peroxidase inhibits the toxic accumulation of $\mathrm{H}_{2} \mathrm{O}_{2}$ in the tissues of the oral cavity [19]. Particularly sensitive to the toxicity of $\mathrm{H}_{2} \mathrm{O}_{2}$ are fibroblasts and epithelial cells, where $\mathrm{H}_{2} \mathrm{O}_{2}$ is converted to hydroxyl radicals, damaging DNA and lipids [16]. $\mathrm{H}_{2} \mathrm{O}_{2}$ can cause oxidative decarboxylation of sialic acid, which interferes with salivary glycoproteins agglutination. Adhering to hydroxyapatite, peroxidase may in turn inhibit bacterial adhesion to it and its subsequent destruction [15, 23]. Other mechanisms of antibacterial OPO are: inhibiting the production of acids by bacteria, inhibiting the transport of glucose and amino acids into the bacteria, and bacterial cell wall damage [22]. Peroxidase catalyzes the oxidation of organic compounds, particularly phenols, which have antibacterial activity (by inhibiting the growth, uptake of glucose and fatty acid production), antifungal and anti-viral [16]. By the oxidation of organic compounds such as phenols, OPO prevents their mutagenic effect in the tissues [24].

The aim of this study was to determine the effect of chronic alcohol intoxication and smoking on the activity of oral peroxidase in saliva.

\section{Material and methods}

Subjects. A total of 37 volunteers participated in the study. This cohort consisted of 17 male alcohol-dependent smoking patients admitted to the Detoxification Unit in Choroszcz after chronic alcohol intoxication (the 'AS' group, standing for alcohol + smoking) (mean age: 42 years; range: 26-55) (100-700 g/day of alcohol; 10-20 cigarettes/day) and 20 control male social drinkers (the 'CNS' group, standing for control non-smokers) with no history of alcohol abuse or smoking (mean age: 42 years; range: 30-53). Alcoholics met criteria for alcohol and nicotine dependence according to ICD-10 (the average time of dependence was $15 \pm 7$ years for alcohol and $20 \pm 8$ years for smoking). The length of the alcohol intoxication ranged from three to 90 days (mean 30). The interview about smoking habit was conducted during a dental examination. Material from persons admitted to the Detoxification Unit was collected on the second day of the abstinence period.

Ethical issues. This study was approved by the Bioethical Committee of the Medical University of Bialystok, Poland (R-I-003/289/2005). Informed written consent was obtained from all the subjects after explanation of the nature, purpose, and potential risks of the study.

Data and sample collection. A check-up of the oral cavity was done by one qualified dentist in artificial light, using a dental mirror and a probe. Following the World Health 
Organization criteria, the level of dental caries in subjects were determined using the DMFT index (decayed, missing or filled teeth; [5]). Gingival status was assessed using the gingival index (GI; Löe and Silness [25]) and the papilla bleeding index (PBI; [26]). The DMFT, GI, and PBI indices of our subjects were: in alcohol-dependent subjects $(19.5 \pm 5.7,0.99 \pm 0.76,0.57 \pm 0.27$, respectively), in controls $(18.8 \pm 5.70,0.30 \pm 0.47,0.35 \pm$ \pm 0.48 , respectively). The subjects were instructed to refrain from smoking, food and beverages, except water, for two hours before saliva collection. All salivary samples ( $3 \mathrm{ml}$ of resting whole saliva) were collected to plastic tubes on ice by the spitting method, under standardized conditions [27, 28], between 8.00 am and 9.00 am to minimize the influence of circadian rhythms. The samples were centrifuged at $3,000 \times \mathrm{g}$ for 20 minutes at $4^{\circ} \mathrm{C}$, to remove cells and debris. The resulting supernatants were divided into $200 \mu \mathrm{l}$ portions, frozen and kept at $-80^{\circ} \mathrm{C}$, until analyzed. Salivary flow (SF) was calculated by dividing the volume of saliva by the time of its collection. In all the samples, peroxidase activity was assayed in duplicate, and the means of the duplicate results were used as final values.

Peroxidase activity assay. Salivary peroxidase activity was measured by colorimetric 2-nitrobenzoic acid-thiocyanate assay [29]. 5,5'-dithiobis-2-nitrobenzoic acid in phosphate buffer ( $\mathrm{pH}$ 5.6) was reduced to nitrobenzoic acid by the addition of mercaptoethanol. The disappearance of nitrobenzoic acid while reacting with OSCN-, the product of peroxidase activity, was analyzed spectrophotometrically at a wavelength of $412 \mathrm{~nm}$. Results were analyzed using the KC Junior from Bio-Tek Instruments.

Statistical analysis. Statistical analysis was performed with Statistica version 8.0 (Statsoft, Krakow, Poland). Results are expressed as means \pm SD. The differences between groups were evaluated using the Mann-Whitney U test. Statistical significance was assumed when $\mathrm{p}<0.05$.

\section{Results}

Compared to the activity of OPO in the control group $(102.4 \pm 29.9 \mathrm{IU} / \mathrm{l}$; mean $\pm \mathrm{SD})$, the activity of OPO in the saliva of smoking alcohol-dependent persons (182.4 \pm 54.0) was significantly higher $(\mathrm{p}=0.00001)$ (Figure 1). Salivary flow (SF) in the control group $(0.43 \pm 0.10 \mathrm{ml} / \mathrm{min})$ was significantly higher than in smoking alcohol-dependent persons $(0.31 \pm 0.31)(p=0.007)$. We found significantly higher GI in smoking alcohol-dependent persons $(0.99 \pm 0.76)$ than in the control group $(0.30 \pm 0.47)$ $(\mathrm{p}=0.003)$ but didn't find significant differences between controls and alcoholics in the PBI or DMFT indices. There were no significant correlations be- tween SF or OPO activity and the number of cigarettes smoked per day or the duration of nicotine dependence. A correlation was found between OPO activity and the number of days of the last alcohol intoxication $\left(\mathrm{r}^{2}=0.55, \mathrm{p}=0.01\right)$ (Figure 2), as well as between OPO activity and gingival in$\operatorname{dex}\left(\mathrm{r}^{2}=0.37, \mathrm{p}=0.04\right)$. There was a correlation between the duration of alcohol dependence and the number of cigarettes smoked per day $\left(r^{2}=0.54\right.$, $\mathrm{p}=0.04)$. OPO salivary values have $70 \%$ sensitivity and $95 \%$ specificity in terms of differentiating between smoking alcoholics and the control group.

\section{Discussion}

It has been reported that chronic ethanol consumption leads to fat accumulation in salivary glands, acinar cell swelling, gland atrophy, as well as to reduced

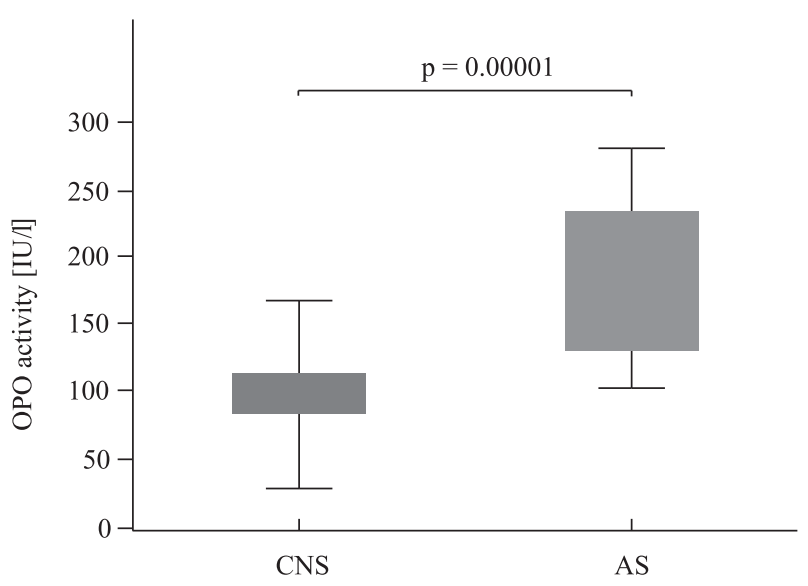

Figure 1. Oral peroxidase (OPO) activity in saliva of control non-smokers (CNS) and smoking alcohol-dependent persons (AS)

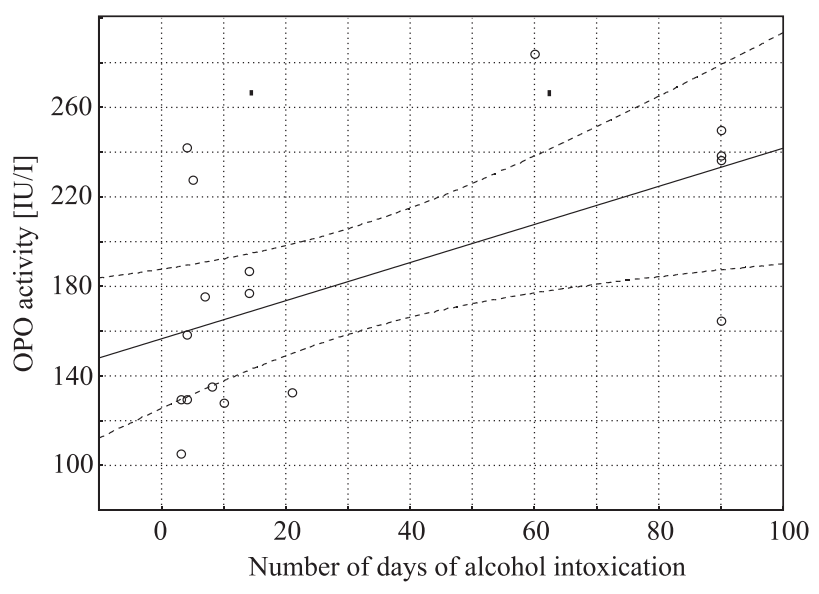

Figure 2. Correlation between oral peroxidase (OPO) activity and number of days of last alcoholic intoxication $\left(\mathrm{r}^{2}=0.55, \mathrm{p}=0.01\right)$ 
salivary flow rate, and markedly decreased salivary protein and glycoprotein levels [6, 30, 31]. A diffuse infiltration of salivary gland stroma by mononuclear inflammatory cells or their concentration around the salivary ducts has also been reported [32]. Ethanol with its own water competition mechanism, acetaldehyde, generated ROS, and non-oxidative metabolites of ethanol (e.g. FAEEs), may all be involved in the toxic effects of alcohol on the salivary glands [2]. In particular, the highly reactive acetaldehyde is capable of forming adducts with (glyco)proteins. It causes morphological changes of the Golgi complex and leads to a general reduction in the efficiency of the secretory process by inhibiting tubulin polymerization. Induced CYP2E1 generates reactive oxygen species (ROS) such as superoxide radical, hydrogen peroxide, etc. ROS can promote conformational changes of proteins by protein oxidation; they can also inactivate enzymes, degrade proteoglycans, cause oxidative damage to the DNA, and damage cellular membranes. The markedly limited cell energy metabolism and ATP availability, while ethanol oxidates to acetaldehyde, are due to the elevated NADH/NAD ratio. It reduces carbohydrate and lipid metabolism and finally causes fatty acids synthesis. Incorporated into membranes, FAEEs have been shown to cause an increase in membrane fluidity. Thus, a disorder in the membrane bilayer and an increase in the lysosomal fragility develop. Membranes are an immediate site of ethanol action. Ethanol and water compete with each other on target membrane molecules. Glycoproteins attract a large volume of water (up to 95\%). Thus displacement of water by ethanol from hydrogen-bonded sites creates the opportunity for allosteric changes that lead to conformational changes of salivary glycoconjugates [33-36].

As with alcohol, cigarette smoke is a source of oxidative stress and high concentrations of acetaldehyde in the oral cavity [10, 37]. It has been proven that concomitant alcohol abuse and cigarette smoking cause a synergistic increase in the concentration of acetaldehyde in saliva [10]. The level of acetaldehyde in the saliva of smokers is double the level in non-smokers [38]. Alcohol abuse and smoking have a synergistic effect on damage to the oral tissues and on tumor development $[10,38]$. However, alcohol abuse carries a more than three times greater risk of developing cancer than smoking [38]. Alcohol abuse has been found to swell secretory cells of the salivary glands, decrease the secretion of proteins, and impair immune mechanisms such as phagocytosis and chemotaxis, as well as increase the production of ROS in smokers [11, 39-41]. Acetaldehyde of cigarette smoke may inactivate peroxidase [14]. Even in people chewing tobacco and using snuff, decreased lev- els of lysozyme and lactoferrin, and increased secretion of immunoglobulin A in the saliva, have been found [15]. In smoking chronic alcohol abusers, lower salivary protein secretion was accompanied by a reduction in the level of amylase, compared to the control group [15]. The significantly higher stimulated secretion of saliva in smokers proves the irritating effect of tobacco smoke on the oral mucosa $[6,39]$.

In chronically intoxicated with alcohol smokers, unstimulated salivary flow (SF) was significantly lower than SF in the control group. Our results confirm the data in the literature [42,43], in which chronic alcohol consumption caused a reduction in SF. Morphological changes of salivary glands, such as those described above, are considered to be involved in SF reduction in people addicted to alcohol. Ethanol, by replacing water, increases the liquidity of cell membranes of the salivary gland, thus impairing the activation of cAMP in the membrane and the mobilization of $\mathrm{Ca}^{2+}$, and leading to a subsequent decrease in secretory cell function. Other mechanisms that may be involved in the SF decrease during abstinence syndrome are: severe stress with sympathetic system arousal, hypercortisolemia, a fluid diet and a smaller number of meals (by reducing the chewing that stimulates saliva secretion), a reduction in the amount of muscarinic receptors, or changes in the secretion of paracrine transmitters and gastrointestinal hormones such as somatostatin, PP peptide, or cholecystokinin [44-49]. As significantly higher secretion of stimulated saliva has been found in smokers [39], the reduction of SF in alcohol-dependent smokers in our study seems to confirm the greater impact of alcohol poisoning than tobacco smoking on the secretion of saliva. The increased activity of OPO does not seem to be the effect of reducing SF alone, because we noticed no inverse correlation between these parameters.

Oxidative stress is very harmful to the tissues and occurs in acute [50] as well as in chronic alcohol intoxication [51]. Oxidative stress is accompanied by the generation of reactive oxygen species (ROS) and increased activity of antioxidant enzymes e.g. glutathione peroxidase $[50,52,53]$. In acute poisoning, ethanol and its metabolites, such as cetaldehyde, may contribute to the inactivation of OPO $[5,14,54]$, a significant oxidative stress in chronic alcohol intoxication is supposed to be compensated by adequate overproduction of antioxidant enzymes. In our study, we observed a significant increase in the activity of OPO after chronic alcohol intoxication in relation to the control group (Figure 1). The increase in the activity of OPO in chronic ethanol intoxication may also be a result of an influx of leukocytes into damaged oral 
mucosa. The involvement of oral tissue damage in OPO increase seems to be confirmed by a significantly higher gingival index (GI) of smoking alcoholics than that of controls, and a significant correlation between the activity of OPO and GI. It is highly likely that the increased activity of OPO that is due to the damage to the oral cavity is mainly due to ethanol intoxication.

In our study, the activity of OPO significantly correlated with the number of days of alcohol intoxication $\left(\mathrm{r}^{2}=0.55, \mathrm{p}=0.01\right)$ (Figure 2). There were no significant correlations between the number of cigarettes smoked per day or the duration of nicotine dependence, indicators of the oral damage (DMFT, PBI, GI), and the activity of OPO.

In conclusion, the increased activity of oral peroxidase in alcohol-dependent smoking patients suggests chronic oxidative stress likely due to ethanol action. Chronically intoxicated alcoholics have a worse gingival status than controls. There is no significant influence of smoking on OPO activity. As OPO has quite good sensitivity and high specificity, it may help in the diagnosis of chronic alcohol abuse.

\section{References}

1. Waszkiewicz N, Szajda SD, Kępka A, Szulc A, Zwierz K. Glycoconjugates in the detection of alcohol abuse. Biochem Soc Trans. 2011;39:365-369.

2. Waszkiewicz N, Szajda SD, Zalewska A et al. Alcohol abuse and glycoconjugate metabolism. Folia Histochem Cytobiol. 2012;50:1-11.

3. Waszkiewicz N, Konarzewska B, Waszkiewicz M et al. Biomarkers of alcohol abuse. Part I. Traditional biomarkers and their interpretation. Psychiatr Pol. 2010;44:127-136.

4. Waszkiewicz N, Popławska R, Konarzewska B et al. Biomarkers of alcohol abuse. Part II. New biomarkers and their interpretation. Psychiatr Pol. 2010;44:137-146.

5. Waszkiewicz N, Szajda SD, Jankowska A et al. The effect of acute ethanol intoxication on salivary proteins of innate and adaptive immunity. Alcohol Clin Exp Res. 2008;32:652-656.

6. Waszkiewicz N, Zalewska A, Szulc A et al. The influence of alcohol on the oral cavity, salivary glands and saliva. Pol Merkur Lekarski. 2011;30:69-74.

7. Waszkiewicz N, Szulc A. Diagnosis of alcohol abuse. Przegl Lek. 2009;66:529-534.

8. Waszkiewicz N, Szulc A. Can we better prevent binge drinking? J Epidemiol Community Health. 2009;63:589.

9. Waszkiewicz N, Szajda SD, Konarzewska B, Szulc A, Kepka A, Zwierz K. Underappreciated role of binge drinking in the risk of lung cancer. Eur J Public Health. 2010;20:6.

10. Salaspuro V, Salaspuro M. Synergistic effect of alcohol drinking and smoking on in vivo acetaldehyde concentration in saliva. Int J Cancer. 2004;111:480-483.

11. Ryder MI. The influence of smoking on host responses in periodontal infections. Periodontol 2000. 2007;43:267-277.

12. Ide R, Mizoue T, Fujino Y et al. Cigarette smoking, alcohol drinking, and oral and pharyngeal cancer mortality in Japan. Oral Dis. 2008;14:314-319.

13. Harrison EL, Desai RA, McKee SA. Nondaily smoking and alcohol use, hazardous drinking, and alcohol diagnoses among young adults: findings from the NESARC. Alcohol Clin Exp Res. 2008;32:2081-2087.

14. Reznick AZ, Klein I, Eiserich JP, Cross CE, Nagler RM. Inhibition of oral peroxidase activity by cigarette smoke: in vivo and in vitro studies. Free Radic Biol Med. 2003;34:377-384.

15. Rudney JD. Does variability in salivary protein concentrations influence oral microbial ecology and oral health? Crit Rev Oral Biol Med. 1995;6:343-367.

16. Ihalin R, Loimaranta V, Tenovuo J. Origin, structure, and biological activities of peroxidases in human saliva. Arch Biochem Biophys. 2006;445:261-268.

17. Amerongen AV, Veerman EC. Saliva - the defender of the oral cavity. Oral Dis. 2002;8:12-22.

18. Klein I, Nagler RM, Toffler R, van Der Vliet A, Reznick AZ. Effect of cigarette smoke on oral peroxidase activity in human saliva: role of hydrogen cyanide. Free Radic Biol Med. 2003;35:1448-1452.

19. Tenovuo J. Antimicrobial agents in saliva - protection for the whole body. J Dent Res. 2002;81:807-809.

20. O'Brien PJ. Peroxidases. Chem Biol Interact. 2000;129:113-139.

21. Marcotte H, Lavoie MC. Oral microbial ecology and the role of salivary immunoglobulin A. Microbiol Mol Biol Rev. 1998;62:71-109.

22. Tenovuo J. Clinical applications of antimicrobial host proteins lactoperoxidase, lysozyme and lactoferrin in xerostomia: efficacy and safety. Oral Dis. 2002;8:23-29.

23. Rosin M, Hanschke M, Splieth C, Kramer A. Activities of lysozyme and salivary peroxidase in unstimulated whole saliva in relation to plaque and gingivitis scores in healthy young males. Clin Oral Investig. 1999;3:133-137.

24. Tenovuo J, Pruitt KM. Relationship of the human salivary peroxidase system to oral health. J Oral Pathol. 1984;13:573-584.

25. Loe H, Silness J. Periodontal disease in pregnancy. I. Prevalence and severity. Acta Odontol Scand. 1963;21:533-551.

26. Saxer UP, Muhlemann HR. Motivation and education. SSO Schweiz Monatsschr Zahnheilkd. 1975;85:905-919.

27. Dawes C. Physiological factors affecting salivary flow rate, oral sugar clearance, and the sensation of dry mouth in man. J Dent Res. 1987;66:648-653.

28. Navazesh M, Christensen C, Brightman V. Clinical criteria for the diagnosis of salivary gland hypofunction. J Dent Res. 1992;71:1363-1369.

29. Mansson-Rahemtulla B, Baldone DC, Pruitt KM, Rahemtulla F. Specific assays for peroxidases in human saliva. Arch Oral Biol. 1986;31:661-668.

30. Riedel F, Goessler U, Hormann K. Alcohol-related diseases of the mouth and throat. Best Pract Res Clin Gastroenterol. 2003; 17:543-555.

31. Slomiany BL, Piotrowski J, Slomiany A. Chronic alcohol ingestion enhances tumor necrosis factor-alpha expression and salivary gland apoptosis. Alcohol Clin Exp Res. 1997;21:1530-1533.

32. Smith PF, Ramirez JC, Romo RE, Esguep AS. Salivary glands in alcoholism: a histologic and sialographic study. Addict Biol. 1996;1:385-393.

33. Waszkiewicz N, Szajda SD, Jankowska A et al. Catabolism of salivary glycoconjugates in acute ethanol intoxication. Med Sci Monit. 2009;15:CR413-CR417.

34. Waszkiewicz N, Szajda SD, Zalewska A et al. Binge drinking-induced liver injury. Hepatology. 2009;50:1676.

35. Waszkiewicz N, Szajda SD, Jankowska A et al. The effect of the binge drinking session on the activity of salivary, serum and urinary $\beta$-hexosaminidase: preliminary data. Alcohol Alcohol. 2008;43:446-450.

36. Waszkiewicz N, Szulc A. Immunity defects in acute and chronic alcohol intoxication. Pol Merkur Lekarski. 2010;29:269-273. 
37. Tapiero H. Influence of alcohol consumption and smoking habits on human health. Biomed Pharmacother. 2004;58:75-76.

38. Seitz HK, Stickel F. Molecular mechanisms of alcohol-mediated carcinogenesis. Nat Rev Cancer. 2007;7:599-612.

39. de Almeida Pdel V, Gregio AM, Machado MA, de Lima AA, Azevedo LR. Saliva composition and functions: a comprehensive review. J Contemp Dent Pract. 2008;9:72-80.

40. Tsoumakidou M, Elston W, Zhu J et al. Cigarette smoking alters bronchial mucosal immunity in asthma. Am J Respir Crit Care Med. 2007;175:919-925.

41. Johnson GK, Guthmiller JM. The impact of cigarette smoking on periodontal disease and treatment. Periodontol 2000. 2007;44:178-194.

42. Dutta SK, Orestes M, Vengulekur S, Kwo P. Ethanol and human saliva: effect of chronic alcoholism on flow rate, composition, and epidermal growth factor. Am J Gastroenterol. 1992;87:350-354.

43. Maier H, Born IA, Veith S, Adler D, Seitz HK. The effect of chronic ethanol consumption on salivary gland morphology and function in the rat. Alcohol Clin Exp Res. 1986;10:425$-427$.

44. De Witte P, Pinto E, Ansseau M, Verbanck P. Alcohol and withdrawal: from animal research to clinical issues. Neurosci Biobehav Rev. 2003;27:189-197.

45. Dodds MW, Johnson DA, Yeh CK. Health benefits of saliva: a review. J Dent. 2005;33:223-233.

46. Jankowska AK, Waszkiel D, Kowalczyk A. Ślina jako główny składnik ekosystemu jamy ustnej. Część I. Mechanizm wydzielania i funkcje. Wiad Lek. 2007;60:148-154.
47. Karolewska E, Konopka T, Pupek M, Chybicka A, Mendak M. Antibacterial potential of saliva in children with leukemia. Oral Surg Oral Med Oral Pathol Oral Radiol Endod. 2008;105:739-744.

48. Keedwell PA, Poon L, Papadopoulos AS, Marshall EJ, Checkley SA. Salivary cortisol measurements during a medically assisted alcohol withdrawal. Addict Biol. 2001;6:247-256.

49. Niebergall-Roth E, Harder H, Singer MV. A review: acute and chronic effects of ethanol and alcoholic beverages on the pancreatic exocrine secretion in vivo and in vitro. Alcohol Clin Exp Res. 1998;22:1570-1583.

50. Scott RB, Reddy KS, Husain K, Schlorff EC, Rybak LP, Somani SM. Dose response of ethanol on antioxidant defense system of liver, lung, and kidney in rat. Pathophysiology. 2000;7:25-32.

51. Campos SC, Moreira DA, Nunes TD, Colepicolo P, Brigagao MR. Oxidative stress in alcohol-induced rat parotid sialadenosis. Arch Oral Biol. 2005;50:661-668

52. Bautista AP. The role of Kupffer cells and reactive oxygen species in hepatic injury during acute and chronic alcohol intoxication. Alcohol Clin Exp Res. 1998;22:255S-259S.

53. Rodrigo R, Trujillo S, Bosco C, Orellana M, Thielemann L, Araya J. Changes in $(\mathrm{Na}+\mathrm{K})$-adenosine triphosphatase activity and ultrastructure of lung and kidney associated with oxidative stress induced by acute ethanol intoxication. Chest. 2002;121:589-596.

54. Waszkiewicz N, Zalewska-Szajda B, Zalewska A et al. Decrease in salivary lactoferrin output in chronically intoxicated alcohol-dependent patients. Folia Histochem Cytobiol. 2012;50:248-254. 